\title{
A MODULUS FOR CURVES FROM DISTANCE
}

\author{
Neil N. Katz \\ New York City College of Technology, Mathematics Department \\ 300 Jay Street, Brooklyn, NY 11201, U.S.A.; nkatz@citytech.cuny.edu
}

\begin{abstract}
In this paper a modulus of curves is defined using pseudo-distance functions. This leads to a notion of quasiconformal maps that is equivalent to the standard definition when the distance function is Riemannian. The moduli of families of curves whose endpoints lie in the boundary of open subsets of a compact, convex set are determined. This allows bounds on volumes of images of Euclidean balls under quasiconformal maps to be made. Also, certain generalized, conformal, isosystolic constants are found. Estimates are given of how these constants, and of how norms of weak upper gradients, vary under quasiconformal maps.
\end{abstract}

\section{Introduction}

In this paper a modulus or outer measure of a set of curves is defined using pseudo-distance functions (see Section 1 for definitions). This does not give a conformal invariant, but does yield a maximal dilation that determines how far a map is from being conformal. In fact, it agrees with the maximal dilation of the conformally invariant (i.e. standard) modulus when the distance function is Riemannian (Theorem 1.10). There are rigidity results when the distance function is given on the boundary of a manifold ([27], [9], [2], [12], and [28]; see also the survey [11] and the monograph [31] and references therein). Other rigidity results have been obtained when the distance function is given outside of a compact, convex subset of a Riemannian manifold ([25], [19] (5.5.C'), and [10]). In light of the equivalence of quasiconformality derived from the modulus of curves from distance to that from the conformally invariant modulus, these results can be seen as analogous to those of Gehring [17], Rešetnjak [29], and Mostow [26].

The paper is organized as follows. The first section begins with definitions and statements of basic properties of the modulus (it also shows how it is related to the standard, conformally invariant modulus). Next is a proof that the maximal dilation of the modulus from a Riemannian distance function agrees with that of the conformally invariant one (Theorem 1.10). This section relies heavily on methods found in the monograph [32].

In the second section estimates of moduli are made, and for certain sets of curves they are determined exactly. In [19] and [10] a compact set whose geodesics are length minimizing up to the boundary is said to be BGM (Boundary Geodesics Minimize). Applying Santalo's formula (as in [19] and [10]) it is shown (Lemma 2.4) that the set

doi:10.5186/aasfm.2010.3501

2000 Mathematics Subject Classification: Primary 30C65, 53C23, 53C65.

Key words: Quasiconformal maps, volume distortion, conformal isosystolic inequalities, conformal filling volume.

This research was partially supported by a grant from the City University of New York PSC-CUNY Research Award Program, the Faculty Fellowship Program of the City University of New York, the Japan Society for the Promotion of Science, Monbusho Grant-in-Aid of Research No. 13088720 and the Graduiertenkolleg of the Mathematisches Insitut, Universität Bonn. 
of curves whose interiors and endpoints lie, respectively, in the interior and boundary of a BGM set $X$ have modulus equal to the Riemannian volume of $X$. More generally, the same conclusion holds (Theorem 2.6) for a subset of a BGM set which is either a countable collection of open, connected sets, or is the complement such a set. For an annulus in Euclidean space, the modulus of the set of curves with one or both endpoints in the inner boundary is also determined (Lemma 2.7).

Calculation of the modulus of the set of curves in Euclidean space with both endpoints in the outer component of the boundary of an annulus (Lemma 2.8) allows a bound on volume distortion to be given for metric balls in Euclidean space under quasiconformal maps that are isometric on the boundary (Theorem 3.1). This result should be compared to (9.6) in [22] where the stronger inequality is stated: $\operatorname{Vol} \varphi(U) \leq \operatorname{Vol}(B)^{\frac{K-1}{K}} \operatorname{Vol}(U)^{\frac{1}{K}}$, where $U \subset B$ are concentric metric balls in Euclidean space and $\varphi: B \rightarrow B$ is $K$-quasiconformal with no constraint on the behaviour of $\varphi$ on the boundary. In dimension two this bound was proved for any measurable set $U$ by K. Astala [3] when $\varphi(0)=0$ as conjectured in [18]. See also [14]. This bound implies one of the form of that in Theorem 3.1 by applying the inequality $u^{t} v^{(1-t)} \leq t u+(1-t) v$ which is well known to hold for any $u, v \geq 0$ and $0<t<1$.

The modulus from pseudo-distance is applicable to the problem of finding minimal volume with distance (or length) bounded below. One example of this is the conformal filling volume. For a compact manifold $V$ of dimension at least two equipped with a distance function $\lambda$, the filling volume is the infimum of volumes among all Riemannian manifolds $(M, g)$ with compact boundary homeomorphic to $V$ such that $\mathrm{d}_{g}(x, y) \geq \lambda(x, y)$ for all $x, y \in V$. This invariant of the boundary was defined by Gromov in [19] where he showed that when $V$ has dimension at least two, the value does not depend on the topology of the manifold $M$. He conjectured that this was also true when $V$ is the circle with Riemannian distance and showed that the minimum area is achieved by the round hemisphere when the bounding surface is a disk. This was extended to the case that the bounding surface is a torus with a disc removed by Bangert, Croke, Ivanov and M. Katz [4]. The question for higher genus surfaces remains open. (In fact the filling volume is unknown for any $V$.) The conformal filling volume $\operatorname{FillVol}(M, \lambda,[g])$ is the infimum of volumes of metrics that satisfy the length condition and are conformal to a fixed metric $g$ on a fixed manifold $M$. This is an approach analogous to the conformal isosystolic inequalities studied by Gromov [19] and by Bavard [5],[6]. (Indeed, isosystolic invariants are only slightly better understood than the filling volume; see the surveys of Berger [7] and Croke and M. Katz [13] or the monograph [23].) Gromov showed that of all metrics conformal to the round, two-dimensional hemisphere (and hence all metrics, since there is only one conformal class) the round hemisphere is the one with minimal area satisfying a lower bound on distance between points on the boundary given by the distance function on the embedded boundary circle. Michèl showed for disks of constant curvature (in the proof of Thèorème $\mathrm{A}$ in [25]), and Croke showed (in the proof of Theorem $\mathrm{C}$ in [10]) that if $(M, g)$ is a compact manifold with boundary of general dimension satisfying a convexity condition, then $g$ has minimal volume among metrics in its conformal class whose distance restricted to $\partial M$ is bounded below by that of $g$. Corollary 3.3 can be seen as a generalization of this last result. 
In Corollary 3.4 estimates are made of changes in the conformal filling volume under quasiconformal maps. The same idea yields a bound on the $L^{n}$ norm of weak upper gradients (in the sense of Heinonen and Koskela [20], who call them very weak gradients) under quasiconformal maps (Corollary 3.2).

Proofs of basic properties stated in the first section are included as an appendix. They are analogous to properties of the conformally invariant modulus and the proofs closely follow those presented in [32]. The appendix is included to give details of their adaptation to the present setting.

Basic facts about Riemannian geometry can be found in [8] and [16].

This paper owes much to the clarity with which Jussi Väisälä wrote [32] and the author would like to thank Werner Ballmann for his suggestion to read it.

\section{A modulus from distance}

Throughout the paper $M, \tilde{M}, M_{1}, M_{2}$ etc will be smooth manifolds of dimension $n \geq 2$ with (possibly empty) boundary under a Riemannian metric denoted by $g, \tilde{g}, g_{1}, g_{2}$ etc. For $X \subset M$ connected and open, $\Omega(X)$ will be the set of all continuous curves in $X$ parameterized on the unit interval. For $Y$ open with closure contained in $X, \Gamma_{Y} \subset \Omega(X)$ are the curves such that $\gamma(0), \gamma(1) \in \partial Y$ and $\gamma(t) \in Y$ for $0<t<1$. Note that if $\partial Y$ has more than one component then endpoints of curves in $\Gamma_{Y}$ could both be in the same component or in different components which is different from the meaning used in [32]. We denote by $\beta_{m}$ the volume of the standard unit sphere of dimension $m$.

Definition 1.1. Let $X$ be a connected, open subset of a Riemannian manifold $(M, g)$, and $\rho: \Omega(X) \rightarrow[0, \infty)$ be invariant under re-parameterization. For all $\mathscr{A} \subset$ $\Omega(X)$, define the $\rho$-admissible functions for $\mathscr{A}$ to be the set of non-negative, Borel measurable functions with values in $[0, \infty]$,

$$
\mathscr{F}_{\rho}(\mathscr{A})=\left\{f \geq 0 \mid \int_{\gamma} f \geq \rho(\gamma) \forall \gamma \in \mathscr{A}\right\}
$$

where the integral is with respect to the length measure on rectifiable curves induced by $g$, and for non-rectifiable curves is by definition infinite unless $f \circ \gamma=0$ almost everywhere, in which case it is zero. Now define the $\rho$-modulus of $\mathscr{A}$

$$
\mathscr{M}_{\rho}(\mathscr{A})=\inf _{f \in \mathscr{F}_{\rho}(\mathscr{A})}\|f\|_{n}^{n}
$$

where $\|\cdot\|_{n}$ denotes the $L^{n}$-norm of the Riemannian measure $d \mu_{g}$ on $X \subset M$ induced by $g$.

For the conformally invariant modulus $\rho(\gamma)=1$ for any curve $\gamma$. This includes constant curves, so the conformally invariant modulus is infinite on sets containing such curves. The $\rho$-modulus is also possibly infinite. However, we have the following lemma.

Lemma 1.2. If $\rho(\gamma)=\lambda(\gamma(0), \gamma(1))$ for $\lambda: X \times X \rightarrow[0, \infty)$ such that $\lambda(x, y) \leq$ $c \mathrm{~d}_{g}(x, y)$ for all $x, y \in X$ and if $\operatorname{Vol}(X, g)$ is finite, then $c \in \mathscr{F}_{\rho}(\Omega(X))$, so that $\mathscr{M}_{\rho}(\mathscr{A})$ is finite for any $\mathscr{A} \subset \Omega(X)$.

Proof. If $\lambda(x, y) \leq c \mathrm{~d}_{g}(x, y)$, then $\int_{\gamma} c \geq c \mathrm{~d}_{g}(\gamma(0), \gamma(1)) \geq \rho(\gamma)$ for any $\gamma \in$ $\Omega(X)$, hence the constant function $c$ is admissible for any $\mathscr{A} \subset \Omega(X)$. The lemma follows immediately from the assumption that $X$ has finite volume. 
Next is an analogue of a theorem of Fuglede ([15] Theorem 1 or [32] Theorem 6.2).

Proposition 1.3. With definitions as above $\mathscr{M}_{\rho}$ defines an outer measure on $\Omega(X)$, i.e.

(i) $\mathscr{M}_{\rho}(\emptyset)=0$,

(ii) if $\mathscr{A}_{1} \subset \mathscr{A}_{2}$, then $\mathscr{M}_{\rho}\left(A_{1}\right) \leq \mathscr{M}_{\rho}\left(A_{2}\right)$, and

(iii) if $\left\{\mathscr{A}_{j} \subset \Omega(X)\right\}_{j \in \mathbf{N}}$, then $\mathscr{M}_{\rho}\left(\cup_{j \in \mathbf{N}} \mathscr{A}_{j}\right) \leq \sum_{j \in \mathbf{N}} \mathscr{M}_{\rho}\left(\mathscr{A}_{j}\right)$.

(For a proof see the appendix.)

It turns out that only rectifiable curves for which $\rho>0$ affect the $\rho$-modulus (c.f. Theorem 6.9 in [32]). Stated another way, non-rectifiable curves as well as those for which $\rho$ is zero have zero $\rho$-modulus. Lemma 1.7 is an analogue to another theorem of Fuglede which also characterizes a family of curves with zero $\rho$-modulus.

Proposition 1.4. For any $\mathscr{A} \subset \Omega(X)$, if $\mathscr{B}=\{\gamma \in \mathscr{A} \mid \gamma$ is rectifiable and $\rho(\gamma)>0\}$, then $\mathscr{M}_{\rho}(\mathscr{A})=\mathscr{M}_{\rho}(\mathscr{B})$.

(See the appendix for a proof.)

Given two manifolds $M, \tilde{M}$, under metrics $g, \tilde{g}$, respectively, connected subsets $X \subset M$ and $\tilde{X} \subset \tilde{M}$, and a homeomorphism $\varphi: X \rightarrow \tilde{X}$, let $\tilde{\rho}(\tilde{\gamma})=\rho\left(\varphi^{-1}(\tilde{\gamma})\right)$, a non-negative function on $\Omega(\tilde{X})$. For any $\mathscr{A} \subset \Omega(X)$, denote $\varphi(\mathscr{A})=\{\varphi \circ \gamma \mid \gamma \in$ $\mathscr{A}\} \subset \Omega(\tilde{X})$.

Definition 1.5. With notation as above define the inner $\rho$-dilation and outer $\rho$-dilation of $\varphi$ to be, respectively,

$$
K_{\rho, I}(\varphi)=\sup \left\{\frac{\mathscr{M}_{\tilde{\rho}}(\varphi(\mathscr{A}))}{\mathscr{M}_{\rho}(\mathscr{A})} \mid \mathscr{A} \subset \Omega(X)\right\}
$$

and

$$
K_{\rho, O}(\varphi)=\sup \left\{\frac{\mathscr{M}_{\rho}(\mathscr{A})}{\mathscr{M}_{\tilde{\rho}}(\varphi(\mathscr{A}))} \mid \mathscr{A} \subset \Omega(X)\right\}
$$

where the suprema are each taken over $\mathscr{A} \subset \Omega(X)$ such that $\mathscr{M}_{\rho}(\mathscr{A})$ and $\mathscr{M}_{\tilde{\rho}}(\varphi(\mathscr{A}))$ are neither both zero nor both infinite, and with the convention that $0 / c=0, c / 0=$ $\infty$ and $\infty / c^{\prime}=\infty$ for any $c \in(0, \infty]$ and $c^{\prime} \in[0, \infty)$. The maximal $\rho$-dilation of $\varphi$ is defined by

$$
K_{\rho}(\varphi)=\max \left\{K_{\rho, I}(\varphi), K_{\rho, O}(\varphi)\right\} .
$$

A map $\varphi$ is $\rho$-quasiconformal iff it has finite maximal $\rho$-dilation and $(K, \rho)$-quasiconformal iff $K_{\rho}(\varphi) \leq K<\infty$.

In this paper, a homeomorphism $\varphi$ will be called quasiconformal if either the maximal dilation of the conformally invariant modulus (i.e. with $\rho \equiv 1$ ) is finite, or if the linear dilation at $x$,

$$
\limsup _{r \rightarrow 0^{+}} \frac{\max \{\mathrm{d}(\varphi(x), \varphi(y)) \mid \mathrm{d}(x, y)=r\}}{\min \{\mathrm{d}(\varphi(x), \varphi(z)) \mid \mathrm{d}(x, z)=r\}}
$$

is bounded independent of $x$. These conditions will be used interchangeably. For a proof that they are equivalent, that they imply $\varphi$ is differentiable almost everywhere, and that $H_{O}\left(\varphi_{*}\right), H_{I}\left(\varphi_{*}\right)$ defined below have finite essential suprema, see [32]. The next proposition is an adaptation of Theorem 13.2 in [32] to the present setting. 
Proposition 1.6. Let $M_{1}, M_{2}, M_{3}$ be Riemannian manifolds and $X_{j} \subset M_{j}$ connected. Fix functions $\rho_{j}$ with $\rho_{3}=\rho_{2} \circ \psi=\rho_{1} \circ \psi \circ \varphi$ and $\rho_{j}: \Omega\left(X_{j}\right) \rightarrow[0, \infty)$ invariant under re-parameterization. If $\varphi: X_{1} \rightarrow X_{2}$ and $\psi: X_{2} \rightarrow X_{3}$ are respectively $\rho_{1}$-quasiconformal and $\rho_{2}$-quasiconformal maps, then the following are true.

(i) $K_{\rho_{2}, I}\left(\varphi^{-1}\right)=K_{\rho_{1}, O}(\varphi)$,

(iv) $K_{\rho_{1}, I}(\psi \circ \varphi) \leq K_{\rho_{2}, I}(\psi) K_{\rho_{1}, I}(\varphi)$,

(ii) $K_{\rho_{2}, O}\left(\varphi^{-1}\right)=K_{\rho_{1}, I}(\varphi)$,

(iii) $K_{\rho_{2}}\left(\varphi^{-1}\right)=K_{\rho_{1}}(\varphi)$,

(v) $K_{\rho_{1}, O}(\psi \circ \varphi) \leq K_{\rho_{2}, O}(\psi) K_{\rho_{1}, O}(\varphi)$,

(vi) $\quad K_{\rho_{1}}(\psi \circ \varphi) \leq K_{\rho_{2}}(\psi) K_{\rho_{1}}(\varphi)$.

(See the appendix for a proof.)

What follows is another theorem of Fuglede adapted to the $\rho$-modulus.

Lemma 1.7. Let $M, \tilde{M}$ be Riemannian manifolds, $X \subset M, \tilde{X} \subset \tilde{M}$ open, connected subsets and $\rho(\gamma)=\lambda(\gamma(0), \gamma(0))$ for all $\gamma \in \Omega(X)$. If $\varphi: X \rightarrow \tilde{X}$ is quasiconformal and $\mathscr{A} \subset \Omega(X)$ is a family of rectifiable curves such that $\varphi \circ \gamma$ is not absolutely continuous for all $\gamma \in \mathscr{A}$, then $\mathscr{M}_{\rho}(\mathscr{A})=0$.

(A proof can be found in the appendix.)

Let $(M, g)$ and $(\tilde{M}, \tilde{g})$ be Riemannian manifolds, $X \subset M, \tilde{X} \subset \tilde{M}$ connected and $\varphi: X \rightarrow \tilde{X}$ differentiable at $p \in X$. If $\operatorname{det}\left(\varphi^{*} \tilde{g}\right)(p) \neq 0$, denote

$$
\begin{aligned}
H_{O}\left(\varphi_{*}\right)(p) & =\frac{\max \left\{\left\|\varphi_{*} v\right\|_{\tilde{g}}^{n} \mid v \in T_{p} M,\|v\|_{g}=1\right\}}{\sqrt{\operatorname{det}\left(\varphi^{*} \tilde{g}\right)(p)}}, \\
H_{I}\left(\varphi_{*}\right)(p) & =\frac{\sqrt{\operatorname{det}\left(\varphi^{*} \tilde{g}\right)(p)}}{\min \left\{\left\|\varphi_{*} v\right\|_{\tilde{g}}^{n} \mid v \in T_{p} M,\|v\|_{g}=1\right\}}, \\
\text { and }\left|\varphi_{*}\right|(p) & =\max \left\{\left\|\varphi_{*} v\right\|_{\tilde{g}} \mid v \in T_{p} M,\|v\|_{g}=1\right\} .
\end{aligned}
$$

If $\operatorname{det}\left(\varphi^{*} \tilde{g}\right)(p)=0$ then define $H_{0}\left(\varphi_{*}\right)(p)=H_{I}\left(\varphi_{*}\right)(p)=\infty$.

Next it is shown that a $K$-quasiconformal homeomorphism is $(K, \rho)$-quasiconformal. The proof closely follows Theorem 15.1 in [32]. It is stated separately because the only assumption required of $\rho$ is invariance under re-parameterization of curves.

Theorem 1.8. With notation as above, if $\varphi: X \rightarrow \tilde{X}$ is a quasiconformal homeomorphism, then

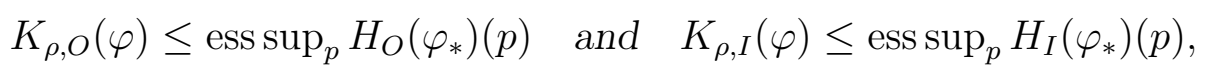

where the suprema are taken over all points $p \in X$ at which $\varphi$ is differentiable. Hence $\varphi$ is $(K, \rho)$-quasiconformal.

Proof. If $K_{\rho, O}(\varphi) \leq \operatorname{ess}_{\sup } H_{O}\left(\varphi_{*}\right)(p)$, then $K_{\rho, I}(\varphi) \leq \operatorname{ess}_{\sup _{p}} H_{I}\left(\varphi_{*}\right)(p)$ follows from Proposition 1.6 (ii) and the fact that $H_{I}\left(\varphi_{*}\right)(p)=H_{O}\left(\varphi_{*}^{-1}\right)(\varphi(p))$ when $\varphi$ is quasiconformal.

If $\operatorname{ess}_{\sup _{p}} H_{O}\left(\varphi_{*}\right)(p)=\infty$, then the inequality is trivial, so we will assume it is finite. Denote

$$
L(\varphi, p)=\limsup _{r \rightarrow 0^{+}} \max \left\{\frac{1}{r} \mathrm{~d}_{\tilde{g}}(\varphi(p), \varphi(q)) \mid \mathrm{d}_{g}(p, q)=r\right\} .
$$

Take $\mathscr{A} \subset \Omega(X)$. If $\mathscr{M}_{\tilde{\rho}}(\varphi(\mathscr{A}))=\infty$, then either $\mathscr{M}_{\rho}(\mathscr{A})=\infty$, in which case $\mathscr{A}$ does not affect the outer $\rho$-dilation, or else it is finite and

$$
\mathscr{M}_{\rho}(\mathscr{A}) \leq \operatorname{ess}_{\sup } H_{O}\left(\varphi_{*}\right)(p) \mathscr{M}_{\tilde{\rho}}(\varphi(\mathscr{A})) \text {. }
$$


If $\mathscr{M}_{\tilde{\rho}}(\varphi(\mathscr{A}))<\infty$, then there exists $\tilde{f} \in \mathscr{F}_{\tilde{\rho}}(\varphi(\mathscr{A}))$ with $\|\tilde{f}\|_{n}<\infty$. Let $f(x)=$ $\tilde{f} \circ \varphi(x) L(\varphi, x)$, and $\mathscr{B} \subset \mathscr{A}$ be those rectifiable curves such that $\varphi \circ \gamma$ is absolutely continuous. Since $\tilde{f} \in \mathscr{F}_{\tilde{\rho}}(\varphi(\mathscr{A}))$, for any $\gamma \in \mathscr{B}$

$$
\int_{\gamma} f=\int_{\gamma} \tilde{f}(\varphi(p)) L(\varphi, p) \geq \int_{\varphi \circ \gamma} \tilde{f} \geq \tilde{\rho}(\varphi \circ \gamma)=\rho(\gamma),
$$

the first inequality following from [32] Theorem 5.3. Therefore $f \in \mathscr{F}_{\rho}(\mathscr{B})$ since $\gamma \in \mathscr{B}$ was arbitrary. Now, $\varphi$ is quasiconformal hence differentiable almost everywhere, so $L(\varphi, p)=\left|\varphi_{*}\right|(p)$ almost everywhere. Applying Lemma 1.7, Proposition 1.3 (iii), and Hölder's inequality,

$$
\begin{aligned}
& \mathscr{M}_{\rho}(\mathscr{A})=\mathscr{M}_{\rho}(\mathscr{B}) \leq\|f\|_{n}^{n}=\int_{X}[\tilde{f} \circ \varphi \cdot L(\varphi, x)]^{n} d \mu_{g} \\
& =\int_{X}[\tilde{f} \circ \varphi]^{n} H_{O}\left(\varphi_{*}\right) \sqrt{\operatorname{det}\left(\varphi^{*} \tilde{g}\right)} d \mu_{g} \\
& \leq \operatorname{ess}_{\sup _{p}} H_{O}\left(\varphi_{*}\right)(p) \int_{X}[\tilde{f} \circ \varphi]^{n} \sqrt{\operatorname{det}\left(\varphi^{*} \tilde{g}\right)} d \mu_{g} \\
& =\operatorname{ess}_{\sup } H_{O}\left(\varphi_{*}\right)(p) \int_{\tilde{X}} \tilde{f}^{n} d \mu_{\tilde{g}}
\end{aligned}
$$

and $\tilde{f} \in \mathscr{F}_{\tilde{\rho}}(\varphi(\mathscr{A}))$ is arbitrary. Therefore, $\mathscr{M}_{\rho}(\mathscr{A}) \leq \operatorname{ess}_{\sup } H_{O}\left(\varphi_{*}\right)(p) \mathscr{M}_{\tilde{\rho}}(\varphi(\mathscr{A}))$.

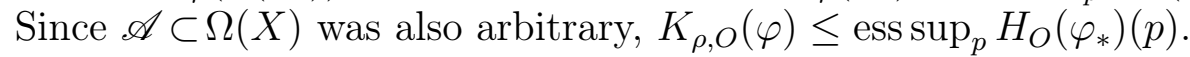

The opposite inequality for the special case that $\rho(\gamma)$ is equal to the Riemannian distance between the endpoints of $\gamma$ is proved separately as Theorem 1.10 below. First we give a lemma whose proof closely follows that of Theorem 15.2 in [32].

Lemma 1.9. With the same notation as in the previous theorem, let $\varphi: X \rightarrow \tilde{X}$ be a homeomorphism that is differentiable at $p_{0} \in X$. If $K_{\rho, O}(\varphi)<\infty$, then

$$
\left|\varphi_{*}\right|^{n}\left(p_{0}\right) \leq K_{\rho, O}(\varphi) \sqrt{\operatorname{det}\left(\varphi^{*} \tilde{g}\right)}\left(p_{0}\right)
$$

where $\rho(\gamma)=\mathrm{d}_{g}(\gamma(0), \gamma(1))$ is the distance function of a Riemannian metric $g$ on $M$.

Proof. Take a unit vector $v_{0} \in T_{p_{0}} M$ such that $a=\left|\varphi_{*}\right|\left(p_{0}\right)=\left\|\varphi_{*}\left(v_{0}\right)\right\|_{\tilde{g}}$. If $a=0$, the conclusion follows since both sides of the inequality are zero. If $a \neq 0$, let $\tilde{v}_{0}=\varphi_{*}\left(v_{0}\right)$.

Take $0<r<\min \left\{\operatorname{inj}_{g}\left(p_{0}\right), \operatorname{inj}_{\tilde{g}}\left(\varphi\left(p_{0}\right)\right), \mathrm{d}_{g}\left(p_{0}, \partial X\right), \mathrm{d}_{\tilde{g}}\left(\varphi\left(p_{0}\right), \partial \tilde{X}\right)\right\}$, and consider Fermi coordinates $\eta: U \rightarrow \mathbf{R}^{n}$ and $\xi: V \rightarrow \mathbf{R}^{n}$ on $M$ to, respectively, the submanifolds $B_{r}\left(p_{0}\right) \cap \exp _{p_{0}}\left(v_{0}^{\perp}\right)$ and $B_{r}\left(\varphi\left(p_{0}\right)\right) \cap \exp _{\varphi\left(p_{0}\right)}\left(\tilde{v}_{0}^{\perp}\right)$ centered at $p_{0}$ and $\varphi\left(p_{0}\right)$. In these coordinates, $\eta_{*}\left(v_{0}\right)=\partial_{1}, \xi_{*}\left(\tilde{v}_{0}\right)=a \partial_{1}$, and $g\left(p_{0}\right), \tilde{g}\left(\varphi\left(p_{0}\right)\right)$ are both diagonal. Fix $0<\varepsilon<a / 2$ and let $Z \subset \mathbf{R}^{n}$ be the closed cube centered at $(\delta / 2,0, \ldots, 0)$ with side length $\delta$ and with faces orthogonal to the coordinate axes. For $\delta \in(0, r)$ sufficiently small, $Z \subset \eta(U), \Phi=\xi \circ \varphi \circ \eta^{-1}: Z \rightarrow \mathbf{R}^{n}$ is well defined and

$$
|\Phi(x)-D \Phi(0)(x)|<\varepsilon \delta
$$


for all $x \in Z$. Let

$$
\begin{aligned}
E & =\left\{x \in Z \mid x_{1}=0\right\}, \\
F & =\left\{x \in Z \mid x_{1}=\delta\right\}, \\
\mathscr{A} & =\left\{\gamma \in \Gamma_{\eta^{-1}\left(Z^{\circ}\right)} \mid \gamma(0) \in \eta^{-1}(E), \gamma(1) \in \eta^{-1}(F), \text { and } \mathrm{d}_{g}(\gamma(0), \gamma(1))=\delta\right\}, \\
\text { and } \tilde{\mathscr{A}} & =\{\varphi \circ \gamma \mid \gamma \in \mathscr{A}\} .
\end{aligned}
$$

By Lemma $2.1 \mathscr{M}_{\rho}(\mathscr{A})$ and $\mathscr{M}_{\tilde{\rho}}(\tilde{\mathscr{A}})$ are finite, where $\tilde{\rho}(\gamma)=\rho\left(\varphi^{-1} \circ \gamma\right)$. Note that $\rho(\gamma)=\delta>0$ for all $\gamma \in \mathscr{A}$, and $\tilde{\rho}(\tilde{\gamma})=\delta>0$ for all $\tilde{\gamma} \in \tilde{\mathscr{A}}$. It follows from (1.6) that $\mathscr{M}_{\rho}(\mathscr{A})>0$. Therefore $\mathscr{M}_{\tilde{\rho}}(\tilde{\mathscr{A}})>0$ as well since $K_{\rho, O}<\infty$ and

$$
K_{\rho, O}(\varphi) \geq \frac{\mathscr{M}_{\rho}(\mathscr{A})}{\mathscr{M}_{\tilde{\rho}}(\tilde{\mathscr{A}})}
$$

Apply Lemma 2.1 to estimate $\mathscr{M}_{\tilde{\rho}}(\tilde{\mathscr{A}})$ and obtain

$$
K_{\rho, O}(\varphi) \geq\left(\frac{\operatorname{Vol}\left(\varphi \circ \eta^{-1}(Z), g\right)}{\tilde{r}^{n}} \delta^{n}\right)^{-1} \mathscr{M}_{\rho}(\mathscr{A})
$$

where $\tilde{r}=\mathrm{d}_{\tilde{g}}\left(\varphi \circ \eta^{-1}(E), \varphi \circ \eta^{-1}(F)\right)$.

Fix $f \in \mathscr{F}_{\rho}(\mathscr{A})$. For every $x=\left(0, x_{2}, \ldots, x_{n}\right) \in E$, let $\gamma_{x}:[0,1] \rightarrow M$ be given by $\gamma_{x}(t)=\eta^{-1}\left(\delta t, x_{2}, \ldots, x_{n}\right)$. By choice of coordinates, $\gamma_{x} \in \mathscr{A}$ is the minimal geodesic realizing the distance from $\gamma_{x}(0) \in \eta^{-1}(E)$ to $\gamma_{x}(1) \in \eta^{-1}(F)$. Hölder's inequality gives that if $f \in \mathscr{F}_{\rho}(\mathscr{A})$,

$$
\rho\left(\gamma_{x}\right)^{n} \leq\left(\int_{\gamma_{x}} f\right)^{n} \leq\left[\left(\int_{\gamma_{x}} 1\right)^{1-1 / n}\left(\int_{\gamma_{x}} f^{n}\right)^{1 / n}\right]^{n}=\delta^{n-1} \int_{\gamma_{x}} f^{n} .
$$

Since $f \in L^{n}(X)$ is non-negative, for almost every $x \in E$,

$$
\begin{aligned}
\left|\int_{\eta \circ \gamma_{x}}\left(f \circ \eta^{-1}\right)^{n} \sqrt{\operatorname{det} g}-\int_{\eta \circ \gamma_{x}}\left(f \circ \eta^{-1}\right)^{n}\right| & \leq \sup _{Z}|\sqrt{\operatorname{det} g}-1| \int_{\eta \circ \gamma_{x}}\left(f \circ \eta^{-1}\right)^{n} \\
& \leq c_{1} \delta \int_{\eta \circ \gamma_{x}}\left(f \circ \eta^{-1}\right)^{n}
\end{aligned}
$$

for $c_{1}>0$ depending on the curvature of $g$ near $p_{0}$ but independent of $\delta$. This estimate is obtained by a standard asymptotic expansion of the pullback of the volume form via the exponential map in terms of Jacobi fields along the curves $\gamma_{x}$. Combining (1.3) and (1.4),

$$
\delta^{1-n} \rho\left(\gamma_{x}\right)^{n} \leq\left(1+c_{2} \delta\right) \int_{\eta \circ \gamma_{x}}\left(f \circ \eta^{-1}\right)^{n} \sqrt{\operatorname{det} g},
$$

where $c_{2}>0$ is also independent of $\delta$. By construction $\rho(\gamma)=\delta$ for all $\gamma \in \mathscr{A}$. Applying this to (1.5) and integrating over all $x \in E$,

$$
\delta \mathrm{Vol}_{n-1}(E) \leq\left(1+c_{2} \delta\right) \int_{X} f^{n} d \mu_{g}
$$


where $\mathrm{Vol}_{n-1}(E)$ is the $(n-1)$-dimensional volume of $E$ as an isometrically immersed submanifold of $(M, g)$. Since $f \in \mathscr{F}_{\rho}(\mathscr{A})$ was arbitrary,

$$
\mathscr{M}_{\rho}(\mathscr{A}) \geq \frac{\delta \mathrm{Vol}_{n-1}(E)}{1+c_{2} \delta} .
$$

Applying (1.4) once more, this time taking $f$ to be $\eta$ composed with the characteristic function of $Z$ which is also in $\mathscr{F}_{\rho}(\mathscr{A})$ and again integrating over $E$,

$$
\operatorname{Vol}\left(\eta^{-1}(Z), g\right) \leq \operatorname{Vol}_{n-1}(E) \delta\left(1+c_{1} \delta\right) .
$$

This, (1.6) and (1.2) imply

$$
K_{\rho, O}(\varphi) \geq \frac{\operatorname{Vol}\left(\eta^{-1}(Z), g\right)(\tilde{r} / \delta)^{n}}{\operatorname{Vol}\left(\varphi \circ \eta^{-1}(Z), \tilde{g}\right)(1+c \delta)^{2}},
$$

where $c=\max \left\{c_{1}, c_{2}\right\}$.

For any $y=\left(y_{1}, \ldots, y_{n}\right) \in \Phi(E),\left|y_{1}\right|<\varepsilon \delta$ by (1.1) and for any $z=\left(z_{1}, \ldots, z_{n}\right) \in$ $\Phi(F),\left|z_{1}-a \delta\right|<\varepsilon \delta$, also by (1.1). Therefore, by the choice of coordinates $(\xi, V)$ on $\tilde{M}$,

$$
\mathrm{d}_{\tilde{g}}(\varphi(p), \varphi(q))>a \delta-2 \varepsilon \delta
$$

for all $p \in \eta^{-1}(E)$ and $q \in \eta^{-1}(F)$. Thus, $\tilde{r} / \delta>a-2 \varepsilon$, the lower bound being positive since $\varepsilon<a / 2$. Combining this with the estimate (1.7) gives

$$
K_{\rho, O}(\varphi) \geq \frac{\operatorname{Vol}\left(\eta^{-1}(Z), g\right)(a-2 \varepsilon)^{n}}{\operatorname{Vol}\left(\varphi \circ \eta^{-1}(Z), g\right)(1+c \delta)^{2}} .
$$
that

Suppose that $\operatorname{det} \varphi^{*} g=0$. Then $\operatorname{ker}\left(\varphi_{*}\right)\left(p_{0}\right)$ is non-empty, which would mean

$$
\operatorname{Vol}\left(\varphi \circ \eta^{-1}(Z), g\right) \leq c_{3} \delta \varepsilon \operatorname{Vol}\left(\eta^{-1}(Z), g\right)
$$

for some constant $c_{3}$ independent of $\delta$, and $\varepsilon$. Since $\varepsilon$ can be chosen arbitrarily small, (1.8) would then imply that $K_{\rho, O}(\varphi)=\infty$ which contradicts the hypothesis. Therefore $\operatorname{det} \varphi^{*} g \neq 0$, and letting $\delta \rightarrow 0$,

$$
\frac{\operatorname{Vol}\left(\varphi \circ \eta^{-1}(Z), g\right)}{\operatorname{Vol}\left(\eta^{-1}(Z), g\right)} \rightarrow \sqrt{\operatorname{det} \varphi^{*} g}\left(p_{0}\right) .
$$

Applying (1.9) to (1.8) for $\delta$ and $\varepsilon$ arbitrarily small, $\sqrt{\operatorname{det} \varphi^{*} g}\left(p_{0}\right) K_{\rho, O}(\varphi) \geq a^{n}$.

Next it is shown that if $\rho(\gamma)$ is the Riemannian distance between the endpoints of $\gamma$, then $\rho$-quasiconformality is equivalent to the definition of a quasiconformality from the conformally invariant modulus.

Theorem 1.10. Let $(M, g)$ and $(\tilde{M}, \tilde{g})$ be Riemannian manifolds, $U \subset M, V \subset$ $\tilde{M}$ open and connected, and $\varphi: U \rightarrow V$ a homeomorphism. If $\rho(\gamma)=\mathrm{d}_{g}(\gamma(0), \gamma(1))$ for a Riemannian metric $g$, then $K_{\rho}(\varphi)=K<\infty$ if and only if $\varphi$ is $K$-quasiconformal.

Proof. Assume that $\varphi$ is $(K, \rho)$-quasiconformal. Quasiconformality will follow from showing that the linear dilation

$$
H\left(x, \varphi^{-1}\right)=\limsup _{r \rightarrow 0} \frac{\max \left\{\mathrm{d}_{g}\left(\varphi^{-1}(x), \varphi^{-1}(y)\right) \mid \mathrm{d}_{g}(x, y)=r\right\}}{\min \left\{\mathrm{d}_{g}\left(\varphi^{-1}(x), \varphi^{-1}(z)\right) \mid \mathrm{d}_{g}(x, z)=r\right\}}
$$

is bounded independent of $x \in V$. Then $\varphi$ is differentiable almost everywhere. From Lemma 1.9 it follows that $\varphi$ is $K$-quasiconformal. 
For $r>0$ smaller than the injectivity radius at $x$, the exponential map based at $x$ is a diffeomorphism of $B_{r}(x)$ with a Euclidean ball. In fact, the exponential map restricted to $B_{r}(x)$ is bi-Lipshitz with constant approaching one as $r$ shrinks to zero. Thus, it is sufficient to prove the special case of when $U$ is a metric ball in $\mathbf{R}^{n}$ and the Riemannian metrics are Euclidean.

Fix $0<r<\mathrm{d}_{g}(x, \partial V)$ and take

$$
L=\max \left\{\mathrm{d}_{g}\left(\varphi^{-1}(x), \varphi^{-1}(y)\right) \mid \mathrm{d}_{g}(x, y)=r\right\}
$$

and

$$
\ell=\min \left\{\mathrm{d}_{g}\left(\varphi^{-1}(x), \varphi^{-1}(z)\right) \mid \mathrm{d}_{g}(x, z)=r\right\} .
$$

Let $N=\left\{y \mid \mathrm{d}_{g}\left(y, \varphi^{-1}(x)\right) \in(\ell, L)\right\}$ and $\mathscr{A} \subset \Gamma_{N}$ be those curves with one endpoint in each component of $\partial N$. Denote $\mathscr{B}=\{\varphi \circ \gamma \mid \gamma \in \mathscr{A}\}, \rho(\gamma)$ the Euclidean distance between the endpoints of $\gamma$, and $\tilde{\rho}(\gamma)=\rho\left(\varphi^{-1}(\gamma)\right)$. We have that $L-\ell \leq \rho(\gamma) \leq L+\ell$ for any $\gamma \in \mathscr{A}$, hence $L-\ell \leq \tilde{\rho}(\gamma) \leq L+\ell$ for all $\gamma \in \mathscr{B}$ as well. If $f \in \mathscr{F}_{\tilde{\rho}}(\mathscr{B})$, then, $\int_{\gamma} \frac{1}{L-\ell} f \geq 1$ for all $\gamma \in \mathscr{B}$, so the conformally invariant modulus of $\mathscr{B}$,

$$
\mathscr{M}(\mathscr{B}) \leq(L-\ell)^{-n} \mathscr{M}_{\tilde{\rho}}(\mathscr{B}) \leq(L-\ell)^{-n} K \mathscr{M}_{\rho}(\mathscr{A}) \leq K \beta_{n-1}|\log L / \ell|^{1-n} .
$$

The last inequality follows by applying Lemma 2.7 with $a=\ell / L$ and stretching by a factor of $L$. This and the estimate of the modulus of $\mathscr{B}$ found in [32] Theorem 11.9 combine to give that

$$
c \leq \mathscr{M}(\mathscr{B}) \leq K \beta_{n-1}(\log (L / \ell))^{1-n},
$$

for constant $c$ that depends only on $n$, and not on $L, \ell, x$ or $r$. From (1.11), in a similar way to the proof in [32] of Theorem 22.3 part (1), $L / \ell$ is bounded by a constant that depends on $K$ and $n$, but not on $r$ or on $x$. Therefore $H\left(x, \varphi^{-1}\right)$ is bounded as desired.

The converse follows from Theorem 1.8.

\section{Modulus estimates}

First is a basic estimate that was used in the proof of Lemma 1.9.

Lemma 2.1. Let $X \subset(M, g)$ be connected and open and $\mathscr{A} \subset \Omega(X)$ such that the images of all curves of $\mathscr{A}$ lie inside a Borel set $U \subset X$. If $L_{g}(\gamma) \geq r>0$ for all $\gamma \in \mathscr{A}$, then

$$
\mathscr{M}_{\rho}(\mathscr{A}) \leq \frac{\operatorname{Vol}(U, g)}{r^{n}}\left(\sup _{\gamma \in \mathscr{A}} \rho(\gamma)\right)^{n}
$$

Proof. Either the right hand side of the estimate is finite, or else it holds trivially. If

$$
f(x)=\frac{1}{r} \chi_{U}(x) \sup _{\gamma \in \mathscr{A}} \rho(\gamma),
$$

then $\int_{\beta} f \geq \sup _{\gamma \in \mathscr{A}} \rho(\gamma)$ for all $\beta \in \mathscr{A}$, so $f \in \mathscr{F}_{\rho}(\mathscr{A})$ and

$$
\mathscr{M}_{\rho}(\mathscr{A}) \leq \frac{1}{r^{n}}\left(\sup _{\gamma \in \mathscr{A}} \rho(\gamma)\right)^{n} \operatorname{Vol}(U, g) .
$$

In the case that $\rho(\gamma)$ is given by the Riemannian distance between the endpoints of $\gamma$, an analogue of Beurling's condition for extremal length [1, Theorem 4-4] can be used to find the $\rho$-modulus of certain families of curves. 
Definition 2.2. A set of curves $\Sigma \subset \Gamma_{X}$ satisfies a Beurling condition for $X \subset$ $M$ under $g$ with respect to $\rho$ iff $L_{g}(\gamma) \geq \rho(\gamma)$ for all $\gamma \in \Gamma_{X}, L_{g}(\gamma)=\rho(\gamma)$ for all $\gamma \in \Sigma$, and if for any Borel function $h$ such that $\int_{\gamma} h \geq 0$ for all $\gamma \in \Sigma$, it follows that $h$ must also satisfy $\int_{X} h d \mu_{g} \geq 0$.

Lemma 2.3. If $\Sigma$ satisfies a Beurling condition on $X$ under $g$ with respect to $\rho$, then

$$
\mathscr{M}_{\rho}\left(\Gamma_{X}\right)=\operatorname{Vol}(X, g) .
$$

Proof. If $f \in \mathscr{F}_{\rho}\left(\Gamma_{X}\right)$, then $\int_{\gamma} f \geq \rho(\gamma)=\int_{\gamma} \chi_{X}$ for all $\gamma \in \Sigma$, so $\int_{X}\left(f-\chi_{X}\right) \geq 0$, and from Hölder's inequality it follows that $\int f^{n} d \mu_{g} \geq \operatorname{Vol}(X, g)$. Since $L_{g}(\gamma) \geq \rho(\gamma)$ for all $\gamma \in \Gamma_{X}, \chi_{X} \in \mathscr{F}_{\rho}\left(\Gamma_{X}\right)$, and so $\mathscr{M}_{\rho}\left(\Gamma_{X}^{M}\right)=\operatorname{Vol}(X, g)$.

Consider an open, pre-compact subset $X$ with smooth boundary in a Riemannian manifold $(M, g)$. For any inward pointing unit tangent vector $v$ not tangent to $\partial X$ let

$$
\tau(v)=\inf \{t>0 \mid \exp (t v) \notin X\} .
$$

After [25],[19],[10], we say that $X$ has the $B G M$ (Boundary Geodesics Minimize) property if $\tau(v)<\infty$ for all inward pointing unit tangent vectors $v$ not tangent to $\partial X$ and if the geodesic $\gamma_{v}:[0, \tau(v)] \rightarrow M$ given by $\gamma_{v}(t)=\exp (t v)$ is minimal.

Lemma 2.4. Let $(M, g)$ be a complete Riemannian $n$-manifold $(n \geq 2), X \subset M$ open with BGM closure, and $\rho(\gamma)=\mathrm{d}_{g}(\gamma(0), \gamma(1))$ for $\gamma \in \Omega(X)$. Then the set of all minimal geodesics with endpoints in $\partial X$ satisfies a Beurling condition for $X$ under $g$ and so $\mathscr{M}_{\rho}\left(\Gamma_{X}\right)=\operatorname{Vol}(X, g)$.

Proof. Let $\Sigma \subset \Gamma_{X}$ be the set of all minimal geodesics with endpoints in $\partial X$. We have that $L_{g}(\gamma)=\rho(\gamma)$ for all $\gamma \in \Sigma$. Let $h$ be a Borel function on $X$ such that $\int_{\gamma} h \geq 0$ for all $\gamma \in \Sigma$. Take $V$ to be the unit tangent vectors to points in $\partial X$ which form an acute angle with the inward-pointing normal. Applying Santalo's formula [30],

$$
\beta_{n-1} \int_{X} h d \mu_{g}=\int_{S X} h(\pi(w)) d \nu(w)=\int_{V} \int_{0}^{\tau(v)} h\left(\exp _{\pi(v)}(t v)\right) d t d \nu(v) \geq 0 .
$$

Here $\nu$ is the measure on the unit tangent bundle $\pi: S \bar{X} \rightarrow \bar{X}$ given by the product of $\mu_{g}$ with the standard measure on the sphere of dimension $n-1$. Thus $\Sigma$ satisfies a Beurling condition for $X$ under $g$ and the conclusion follows from Lemma 2.3.

Next the $\rho$-modulus of a set of curves is bounded above by the sum of $\rho$-moduli of sets of subcurves when $\rho$ is induced by a pseudo distance function $\lambda$.

Lemma 2.5. Let $E$ be a path-connected, open subset of $(M, g)$ and take $\rho(\gamma)=$ $\lambda(\gamma(0), \gamma(1))$ for all $\gamma \in \Omega(E)$, where $\lambda$ is symmetric, bounded on $E \times E$, and obeys the triangle inequality. If $\left\{U_{j}\right\}_{j \in \mathscr{I}}$ is a countable (or finite) collection of disjoint, connected, non-empty, open subsets of $E$ such that $E \backslash \bigcup_{j \in \mathscr{I}} U_{j}$ has zero measure then,

$$
\mathscr{M}_{\rho}\left(\Gamma_{E}\right) \leq \sum_{j \in \mathscr{I}} \mathscr{M}_{\rho}\left(\Gamma_{U_{j}}\right)
$$


Proof. If any $\mathscr{M}_{\rho}\left(\Gamma_{U_{j}}\right)$ is infinite or if their sum is infinite then the upper bound certainly holds, so we can assume the sum converges. By Proposition 1.4, it suffices to show that $\mathscr{M}_{\rho}(\mathscr{A})$ has the given upper bound where $\mathscr{A} \subset \Gamma_{E}$ are all the rectifiable curves in $\Gamma_{E}$. Let $E^{\prime}=E \backslash \bigcup_{j \in \mathscr{I}} U_{j}$, a set of measure zero. Take $u_{j} \in \mathscr{F}_{\rho}\left(\Gamma_{U_{j}}\right)$ with $\operatorname{supp} u_{j} \subset \overline{U_{j}}$. Let $u(x)=\infty$ for all $x \in E^{\prime}$ and $u(x)=0$ otherwise.

For $\gamma \in \mathscr{A}$ parameterized proportional to arc-length and $V \subset \gamma^{-1}\left(U_{j}\right)$ any connected component, denote by $\gamma_{V}$ the re-parameterization of $\left.\gamma\right|_{\bar{V}}$ to $[0,1]$ proportional to arclength. Then $\int_{\gamma_{V}} u_{j} \geq \rho\left(\gamma_{V}\right)$. Now, $\int_{\gamma} u=\infty$ whenever $\gamma^{-1}\left(E^{\prime}\right)$, has non-zero Borel measure on $[0,1]$, so we are left with the case when $\gamma^{-1}\left(E^{\prime}\right)$ has measure zero. Since $[0,1]$ is separable, there are countably many connected components of $\gamma^{-1}\left(U_{j}\right)$, whence $\gamma^{-1}\left(\bigcup_{j \in \mathscr{I}} U_{j}\right)$ has countably many connected components $\left\{V_{\ell}\right\}_{\ell \in \mathbf{N}}$. Denote by $\gamma_{\ell}$ the re-parameterization of $\left.\gamma\right|_{\bar{V}_{\ell}}$ to $[0,1]$ proportional to arclength. Since $\left\{U_{j}\right\}_{j \in \mathscr{I}}$ are disjoint, and $u, u_{1}, u_{2}, \ldots$ are non-negative,

$$
\int_{\gamma}\left(u^{n}+\sum_{j \in \mathscr{I}} u_{j}^{n}\right)^{1 / n} \geq \sum_{\ell \in \mathbf{N}} \int_{\gamma_{\ell}} \sum_{j \in \mathscr{I}} u_{j} \geq \sum_{\ell \in \mathbf{N}} \rho\left(\gamma_{\ell}\right) \geq \rho(\gamma),
$$

the last estimate following from the triangle inequality. Therefore, $\left(u^{n}+\sum_{j \in \mathscr{I}} u_{j}^{n}\right)^{1 / n}$ $\in \mathscr{F}_{\rho}(\mathscr{A})$, and

$$
\mathscr{M}_{\rho}(\mathscr{A}) \leq \int_{M}\left(u^{n}+\sum_{j \in \mathscr{I}} u_{j}^{n}\right) d \mu_{g} \leq \sum_{j \in \mathscr{I}} \int_{M} u_{j}^{n} d \mu_{g} .
$$

Since $u_{j} \in \mathscr{F}_{\rho}\left(\Gamma_{U_{j}}\right)$ were arbitrary, $\mathscr{M}_{\rho}(\mathscr{A}) \leq \sum_{j \in \mathscr{I}} \mathscr{M}_{\rho}\left(\Gamma_{U_{j}}\right)$.

For certain sets the above lemma yields the modulus exactly.

Theorem 2.6. Let $A \subset(M, g)$ be an open, pre-compact subset of a compact Riemannian manifold $M$ of dimension at least two. Fix $B \subset A$ open, compactly contained in $A$ with countably many connected components such that $\partial B$ has measure zero, and denote $B^{\prime}=A^{\circ} \backslash \bar{B}$. If $\rho(\gamma)=\mathrm{d}_{g}(\gamma(0), \gamma(1))$ and $\mathscr{M}_{\rho}(\Sigma)=\operatorname{Vol}(A, g)$ for some $\Sigma \subset \Gamma_{A}$, then

$$
\mathscr{M}_{\rho}\left(\Gamma_{B^{\prime}}\right)=\operatorname{Vol}\left(B^{\prime}, g\right) \text { and } \mathscr{M}_{\rho}\left(\Gamma_{B}\right)=\operatorname{Vol}(B, g) .
$$

Proof. Let $\left\{B_{j}\right\}_{j \in \mathscr{I}}$ be the connected components of $B$. By Lemma 2.5, Lemma 1.2 and Proposition 1.3

$$
\begin{aligned}
\operatorname{Vol}(A, g) & =\mathscr{M}_{\rho}(\Sigma) \leq \mathscr{M}_{\rho}\left(\Gamma_{A}\right) \leq \mathscr{M}_{\rho}\left(\Gamma_{B^{\prime}}\right)+\sum_{j \in \mathscr{I}} \mathscr{M}_{\rho}\left(\Gamma_{B_{j}}\right) \\
& \leq \operatorname{Vol}(A \backslash B, g)+\sum_{j \in \mathscr{I}} \operatorname{Vol}\left(B_{j}, g\right)=\operatorname{Vol}(A, g),
\end{aligned}
$$

so all the inequalities are in fact equalities. In particular, $\mathscr{M}_{\rho}\left(\Gamma_{B^{\prime}}\right)=\operatorname{Vol}(A \backslash B, g)$, and $\mathscr{M}_{\rho}\left(\Gamma_{B}\right)=\operatorname{Vol}(B, g)$.

Next we will consider annuli in Euclidean space. Let $V=B_{1}(0) \backslash \overline{B_{a}(0)} \subset \mathbf{R}^{n}$, with $0<a<1$, and $\mathscr{A}_{j} \subset \Gamma_{V}$ be the set of curves with exactly $j$ endpoints in $\partial \mathrm{B}(1)$. 
Employing a similar extremal function to that used in [32] for spherical rings, we can find the $\rho$-modulus for $\mathscr{A}_{0} \cup \mathscr{A}_{1}$.

Lemma 2.7. With notation as above, if $\rho$ is induced by the Euclidean distance, then

$$
\mathscr{M}_{\rho}\left(\mathscr{A}_{0} \cup \mathscr{A}_{1}\right)=\beta_{n-1} \frac{(1-a)^{n}}{|\log a|^{n-1}} .
$$

Proof. Working in polar co-ordinates, let $f(r, \Theta)=\frac{a-1}{\log a} \cdot \frac{1}{r} \cdot \chi_{\bar{V}}$. We will show that $f$ is admissible for $\mathscr{A}_{0}$ and $\mathscr{A}_{1}$, whence

$$
\mathscr{M}_{\rho}\left(\mathscr{A}_{0} \cup \mathscr{A}_{1}\right) \leq\|f\|_{n}^{n}=\beta_{n-1} \frac{(1-a)^{n}}{|\log a|^{n-1}} .
$$

The reverse inequality is arrived at in much the same way as in [32]. For any $\tilde{f} \in$ $\mathscr{F}_{\rho}\left(\mathscr{A}_{1}\right)$, if $\theta \in S^{n-1}$, then applying Hölder's inequality,

$$
(1-a) \leq \int_{a}^{1} \tilde{f}(t \theta) d t \leq\left(\int_{a}^{1} \tilde{f}(t \theta) \cdot t^{n-1} d t\right)^{1 / n}(-\log a)^{1-1 / n}
$$

taking the $n^{\text {th }}$ power and integrating over $S^{n-1}$ gives that

$$
\|\tilde{f}\|_{n}^{n} \geq \beta_{n-1}(1-a)^{n} /|\log a|^{n-1}
$$

and since $\tilde{f}$ was arbitrary, the result follows.

To show $f \in \mathscr{F}_{\rho}\left(\mathscr{A}_{0}\right)$, consider the stereographic projection $\varphi: V \rightarrow \mathbf{R}^{n+1}$ given by

$$
\varphi(x)=\frac{2 a^{2}}{a^{2}+|x|}\left(x_{1}, \ldots, x_{n}, \frac{a^{2}-|x|^{2}}{2 a}\right),
$$

where $|x|$ is the Euclidean norm of $x$. This maps $V$ to the $n$-sphere of radius $a$ sending the inner boundary to the equator. If we pull back the metric with constant curvature $1 / a^{2}$ via $\varphi$ to $V$, we get a metric conformal to the Euclidean metric with conformal factor

$$
F(r, \Theta)=\frac{2 a^{2}}{a^{2}+r^{2}} \chi_{\bar{V}}
$$

Distances in this metric on the inner boundary of $V$ (i.e. $\left.\partial B_{a}(0)\right)$ are at least as large as the Euclidean distance, whence $F \in \mathscr{F}_{\rho}\left(\mathscr{A}_{0}\right)$. It is elementary to show that $0 \leq F / f \leq 1$ and therefore $f(r, \Theta) \geq F(r, \Theta)$ for all $a \leq r \leq 1$. It immediately follows that $f$ is $\rho$-admissible for $\mathscr{A}_{0}$.

To show that $f$ is $\rho$-admissible for $\mathscr{A}_{1}$, consider the metric $g=f^{2} g_{0}$ where $g_{0}$ is the Euclidean metric. For this it is sufficient to show that $\mathrm{d}_{g}(p, q) \geq \mathrm{d}_{g_{0}}(p, q)$ for all $p \in \partial B_{a}(0)$ and all $q \in \partial B_{1}(0)$. Take the standard co-ordinates $\left(x^{1}, \ldots, x^{n}\right)$ with co-ordinate fields $\partial_{1}, \ldots, \partial_{n}$. Consider the vector fields $Y=\frac{x^{j}}{r} \partial_{j}$ (we use the Einstein convention) and $Z=x^{2} \partial_{1}-x^{1} \partial_{2}$. A straightforward calculation yields that on the 2-plane $\omega=\left\{x \mid x^{3}=\ldots=x^{n}=0\right\},\left.\nabla_{Y} Y\right|_{\omega}=-\left.\frac{1}{r} Y\right|_{\omega}$, and $\left.\nabla_{Z} Z\right|_{\omega}=$ $\nabla_{Y} Z=\nabla_{Z} Y=0$. It follows that this 2-plane is totally geodesic under $g$. Since $g$ is rotationally symmetric, in order to show that $\mathrm{d}_{g}(p, q) \geq \mathrm{d}_{g_{0}}(p, q)$ for all $p \in \partial B_{a}(0)$ and all $q \in \partial B_{1}(0)$, it is sufficient to show this is true when restricted to this same 2-plane.

Consider polar coordinates in this two plane with co-ordinate fields $\zeta_{1}=\partial_{r}=$ $\frac{x_{1}}{r} \partial_{1}+\frac{x_{2}}{r} \partial_{2}$ and $\zeta_{2}=\partial_{\theta}=-x_{2} \partial_{1}+x_{1} \partial_{2}$. In this co-ordinate system the Christoffel 
symbols are all zero except for $\Gamma_{11}^{1}=\left\langle\nabla_{\partial_{r}} \partial_{r}, \partial_{r}\right\rangle_{g} /\left\langle\partial_{r}, \partial_{r}\right\rangle_{g}=-\frac{1}{r}$. If $\gamma:[0,1] \rightarrow V$ is a geodesic with $\gamma(t)=(r(t), \theta(t)), \gamma(0)=(a, 0)$, and $\gamma(1)=(1, \alpha)$, the geodesic equations yield:

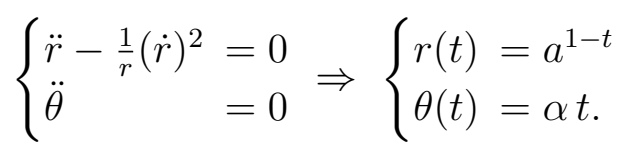

These geodesics have tangent vectors that are never orthogonal to $Y$. The curvature of $g$ can be calculated as follows. For fixed $\lambda \neq \mu$ (so the Einstein convention will not apply to $\lambda$ and $\mu$ ) define $\xi=x^{\lambda} \partial_{\mu}-x^{\mu} \partial_{\lambda}$. Then we have $\nabla_{\xi} Y=\nabla_{Y} \xi=0$, hence $[\xi, Y]=0$ and $\left\langle\nabla_{\xi} \nabla_{Y} \xi, Y\right\rangle_{g}=0$. Also, $\nabla_{\xi} \xi=-x^{\lambda} \partial_{\lambda}-x^{\mu} \partial_{\mu}+\frac{\left(x^{\lambda}\right)^{2}+\left(x^{\mu}\right)^{2}}{r} Y$, and

$$
\begin{aligned}
R(\xi, Y, \xi, Y) & =\nabla_{Y} \nabla_{\xi} \xi, Y_{g}=Y\left\langle\nabla_{\xi} \xi, Y\right\rangle_{g}-\left\langle\nabla_{\xi} \xi, \nabla_{Y} Y\right\rangle \\
& =Y\left\langle\nabla_{\xi} \xi, Y\right\rangle_{g}+\frac{1}{r}\left\langle\nabla_{\xi} \xi, Y\right\rangle=0 .
\end{aligned}
$$

Since $g$ has zero curvature on any tangent plane containing the radial direction, no geodesic $\gamma$ as above can have a conjugate point before the cut distance. Radial symmetry of $g$ gives that $\gamma:[0,1] \rightarrow V$ must be minimizing on $[0,1]$ as long as $-\pi \leq \alpha \leq \pi$.

Integrating gives us the length of $\gamma$ to be $(1-a) \sqrt{1+(\alpha / \log a)^{2}}$. It remains to show that

$$
(a-1)^{2}\left[\frac{\alpha^{2}}{(\log a)^{2}}+1\right] \geq a^{2}+1-2 a \cos \alpha,
$$

where the right hand side of the inequality is the square of the Euclidean distance between $(a, 0)$ and $(1, \alpha)$. Using elementary methods this follows for all $0<a<1$ and $-\pi \leq \alpha \leq \pi$.

Now we will use Beurling's condition to find the $\rho$-modulus of sets of curves with both endpoints in the outer boundary. The method is similar to that found in section 4-9 of [1]). Let $V=B_{1}(0) \backslash \overline{B_{a}\left(x_{0}\right)} \subset \mathbf{R}^{n}$ with $\left|x_{0}\right|+a<1$ and $a>0$. Denote by $\mathscr{A} \subset \Gamma_{V}$ the set of curves with both endpoints in $\partial B_{1}(0)$.

Lemma 2.8. With notation as above, if $\rho$ is induced by the Euclidean distance, then

$$
\mathscr{M}_{\rho}(\mathscr{A})=\operatorname{Vol} V \text {. }
$$

Proof. First we will show that the lemma holds when $x_{0}=0$. Let $\Sigma \subset \mathscr{A}$ be the set of all straight line segments with both endpoints in $\partial B_{1}(0)$ that do not enter $B_{a}(0)$. If we can show that for dimension two $\Sigma$ satisfies a Beurling condition under the Euclidean metric, then (since we are in Euclidean space) by applying Fubini's theorem it follows that the Beurling condition holds for any finite dimension.

Take polar coordinates $(r, \theta)$ and consider the line segment starting at $(1, \theta)$ whose farthest distance to $\partial B_{1}(0)$ is $t$. If $s$ is the arclength of the segment beginning at $(1, \theta)$, then

$$
\begin{aligned}
r^{2} & =1-2 s \sqrt{2 t-t^{2}}+s^{2}, \\
\text { thus } \quad s & =\sqrt{2 t-t^{2}}-\sqrt{2 t-t^{2}+r^{2}-1} \\
\text { and } d s \wedge d \theta & =\frac{1}{\sqrt{r^{2}-(1-t)^{2}}} r d \theta \wedge d r .
\end{aligned}
$$


Take $h$ to be a Borel function such that $\int_{\gamma} h \geq 0$ for all $\gamma \in \Sigma$. Denote by $\chi$ the characteristic function of the interval $[1, \infty)$. Now

$$
\begin{aligned}
\frac{\pi}{2} \int_{a}^{1} \int_{0}^{2 \pi} h(r, \theta) r d \theta d r & =\int_{a}^{1} \int_{0}^{2 \pi} h(r, \theta) r \int_{0}^{1-a} \frac{(1-t) \chi(t /(1-r))}{\sqrt{(1-t)^{2}-a^{2}} \sqrt{r^{2}-(1-t)^{2}}} d t d \theta d r \\
& =\int_{0}^{1-a} \frac{(1-t)}{\sqrt{(1-t)^{2}-a^{2}}} \int_{a}^{1} \int_{0}^{2 \pi} \frac{\chi(t /(1-r)) h(r, \theta)}{\sqrt{r^{2}-(1-t)^{2}}} r d \theta d r d t \\
& =\int_{0}^{1-a} \frac{(1-t)}{\sqrt{(1-t)^{2}-a^{2}}} \int_{0}^{2 \pi} \int_{0}^{2 \sqrt{2 t-t^{2}}} \chi(t /(1-r(s))) h(s, \theta) d s d \theta d t \\
& \geq 0
\end{aligned}
$$

the last inequality holding because if $t$ is the farthest that $\gamma \in \Sigma$ gets from $\partial B_{1}(0)$, then $t \geq 1-r(\gamma)$.

For the general case, let $\tilde{V}=B_{r}\left(x_{0}\right) \backslash \overline{B_{a}\left(x_{0}\right)}$ with $r>\left|x_{0}\right|+1$ and take $\tilde{\Sigma}$ to be all the straight line segments with both endpoints in $\partial B_{r}\left(x_{0}\right)$ that do not enter $B_{a}\left(x_{0}\right)$. If $h: V \rightarrow \mathbf{R}$ is Borel such that $\int_{\gamma} h \geq 0$ for all $\gamma \in \Sigma$. Let $H(x)=h(x)$ for $x \in V$ and $H(x)=0$ for $x \in B_{r}\left(x_{0}\right) \backslash V$. Each $\gamma \in \Sigma$ has a unique extension to $\tilde{\gamma} \in \tilde{\Sigma}$ and $\int_{\tilde{\gamma}} H=\int_{\gamma} h \geq 0$. Furthermore if $\tilde{\gamma} \in \tilde{\Sigma}$ doesn't intersect $V$ then $\int_{\tilde{\gamma}} H=0$. It is easy to see that $\tilde{\Sigma}$ has the Beurling property for $B_{r}\left(x_{0}\right) \backslash \overline{B_{a}\left(x_{0}\right)}$ as these are concentric metric balls. Therefore $\int_{\tilde{V}} H \geq 0$ and $\int_{V} h=\int_{\tilde{V}} H$.

\section{Applications}

First we will apply Theorem 1.8 and the modulus estimate Lemma 2.8 to give a volume bound for quasiconformal maps on the unit Euclidean ball whose restriction to the boundary is an isometry.

Theorem 3.1. Let $\varphi: B_{1}(0) \rightarrow B_{1}(0)$ be a $K$-quasiconformal map on the unit ball in $\mathbf{R}^{n}$ that preserves distance on the boundary. For any Euclidean ball $U=$ $B_{a}(0) \subset B_{1}(0)$,

$$
\begin{aligned}
\operatorname{Vol} \varphi(V) & \geq \frac{1}{K} \operatorname{Vol} V \\
\operatorname{Vol} \varphi(U) & \leq \frac{K-1}{K} \operatorname{Vol} B_{1}(0)+\frac{1}{K} \operatorname{Vol} U
\end{aligned}
$$

where $V=B_{1}(0) \backslash U$.

Proof. Extend $\varphi$ to a map $\Phi: B_{r}(0) \rightarrow B_{r}(0)$ for $r>1$ by letting $\Phi(x)=$ $|x| \varphi(x /|x|)$ for $|x|>1$ and $\Phi(x)=\varphi(x)$ for $|x| \leq 1$. Since $\Phi$ is an isometry on $B_{r}(0) \backslash B_{1}(0)$, and is equal to a $K$-quasiconformal map on $B_{1}(0)$, it must itself be $K$-quasiconformal. We will show that (3.12) holds when $U$ is compactly contained in $B_{1}(0)$. The more general statement follows from $\varphi$ being a homeomorphism. Let $\mathscr{A}$ be as in Lemma 2.8. Applying Lemma 2.8, and Theorem 1.8 with $\rho$ induced by 
the Euclidean distance,

$$
\operatorname{Vol} V=\mathscr{M}_{\rho}(\mathscr{A}) \leq K \mathscr{M}_{\tilde{\rho}}(\Phi(\mathscr{A}))
$$

where $\tilde{\rho}=\rho \circ \Phi^{-1}$. Since $\Phi$ preserves distance on $\partial B_{1}(0), \rho(\Phi(\gamma))=\rho(\gamma)$ for all $\gamma \in \mathscr{A}$. Thus the characteristic function of $\varphi(V)=\Phi(V)$ is $\tilde{\rho}$-admissible for $\varphi(\mathscr{A})$, so $\mathscr{M}_{\tilde{\rho}}(\varphi(\mathscr{A})) \leq \operatorname{Vol} \varphi(V)$. The first estimate follows immediately and the second one from the first and the observation that $\operatorname{Vol} U+\operatorname{Vol} V=\operatorname{Vol} \varphi(U)+\operatorname{Vol} \varphi(V)=$ Vol $B_{1}(0)$.

Next is a bound on the $L^{n}$-norm of weak upper gradients in the sense of Heinonen and Koskela [20]. Let $W$ be a connected open subset of $(M, g)$ and $u$ a real-valued function on $W$. Let $\rho_{u}(\gamma)=|u(\gamma(0))-u(\gamma(1))|$ for $\gamma \in \Omega(W)$. Suppose that there exists a non-negative $L^{n}$ function $f$ on $W$ such that

$$
\int_{\gamma} f \geq \rho_{u}(\gamma)
$$

for all $\gamma \in \Omega(W)$. We will call such an $f$ a weak upper gradient of $u$.

Corollary 3.2. Let $X \subset(M, g), Y \subset(\tilde{M}, \tilde{g})$ be open and connected. If $\psi: Y \rightarrow$ $X$ is $K$-quasiconformal and $u$ is a function on $X$ that has a weak upper gradient with finite $L^{n}$ norm, then so does $u \circ \psi$, and either,

$$
\frac{1}{K} \leq \frac{\inf \left\{\|f\|_{n}^{n} \mid f \text { is a weak upper gradient of } u \circ \psi\right\}}{\inf \left\{\|\tilde{f}\|_{n}^{n} \mid \tilde{f} \text { is a weak upper gradient of u }\right\}} \leq K,
$$

or both infima are zero.

Proof. If we let $\rho=\rho_{\psi \circ u}$ and $\tilde{\rho}(\gamma)=\rho_{u}$ as above, then either both infima are zero, or else since

$$
\inf \left\{\|\tilde{f}\|_{n}^{n} \mid \tilde{f} \text { is a weak upper gradient of } u\right\}=\mathscr{M}_{\tilde{\rho}}(\Omega(X))=\mathscr{M}_{\tilde{\rho}}(\Omega(\varphi(Y)))
$$

and $\inf \left\{\|f\|_{n}^{n} \mid f\right.$ is a weak upper gradient of $\left.u \circ \psi\right\}=\mathscr{M}_{\rho}(\Omega(Y))$,

the conclusion follows from Theorem 1.8.

Next we apply Theorem 2.6 to calculate conformal filling volume and then use Theorem 1.8 to obtain estimates for conformal filling volume. The moduli we determined in the previous section yield the conformal filling volume in a broad range of cases that can be seen as generalizations of the conformal filling volume of the 2-disc in [19].

Corollary 3.3. Let $(M, g)$ be a compact, BGM, Riemannian manifold with boundary with dimension $\operatorname{dim}(M)=n \geq 2$ and $V$ open and compactly contained in $M$ with countably many components.

(i) If $h$ is any metric on $M \backslash V$ conformal to $\left.g\right|_{M \backslash V}$ such that $\mathrm{d}_{h}(x, y) \geq \mathrm{d}_{g}(x, y)$ for all $x, y \in \partial(M \backslash V)$, then $\operatorname{Vol}(M \backslash V, h) \geq \operatorname{Vol}(M \backslash V, g)$.

(ii) If $V$ is connected and $h$ is any metric on $V$ conformal to $\left.g\right|_{V}$ such that $\mathrm{d}_{h}(x, y) \geq \mathrm{d}_{g}(x, y)$ for all $x, y \in \partial V$, then $\operatorname{Vol}(V, h) \geq \operatorname{Vol}(V, g)$.

Proof. Let $\rho(\gamma)=\mathrm{d}_{g}(\gamma(0), \gamma(1))$ for $\gamma \in \Omega(M)$. Lemma 2.4 and Theorem 2.6 together show that the minimal $L^{n}$ norm among $\rho$-admissible $L^{n}$ functions for $\Gamma_{V}$ and $\Gamma_{M \backslash \bar{V}}$ is realized by the constant function 1 . Therefore the minimal $L^{n}$ norm among continuous, $\rho$-admissible functions (and hence minimal volume) is also realized by this same function. 
In [24] it was shown that a necessary and sufficient condition for conformal filling volume to be realized by a continuous, positive function was the existence of a set of curves satisfying a weaker Beurling condition (on continuous, rather than Borel functions). In this case it can be shown that when $V$ is compactly contained in $M$ the set of curves in question are the geodesics with endpoints in the boundary of $V$ and interiors in $V$.

Now we will apply Theorem 1.8 to give bounds on how conformal filling volume varies under a quasiconformal map. This can be seen as a weak analogue to Wolpert's Theorem on the length of homotopy classes of closed geodesics in the hyperbolic metric.

Corollary 3.4. Let $M$ be a compact Riemannian manifold with boundary of dimension at least two, and $g, \tilde{g}$ smooth metrics on $M$. If $(M, g)$ is BGM and there exists a $K$-quasiconformal map $\psi:(M, g) \rightarrow(M, \tilde{g})$, then the conformal filling volumes satisfy

$$
\operatorname{Fill} \operatorname{Vol}(M, \lambda,[\tilde{g}]) \geq \frac{1}{K} \operatorname{Vol}(M, g),
$$

where $\lambda(x, y)=\mathrm{d}_{g}\left(\psi^{-1}(x), \psi^{-1}(y)\right)$.

Proof. Let $\rho(\gamma)=\mathrm{d}_{g}\left((\gamma(0), \gamma(1))\right.$ and $\tilde{\rho}(\gamma)=\rho\left(\psi^{-1} \circ \gamma\right)=\lambda(\gamma(0), \gamma(1))$. Applying Corollary 3.3 and the arguments in Theorem 1.8,

$$
\operatorname{Vol}(M, g)=\operatorname{FillVol}\left(M, \mathrm{~d}_{g},[g]\right)=\mathscr{M}_{\rho}(\rho) \Gamma_{M} \leq K \mathscr{M}_{\tilde{\rho}}\left(\Gamma_{M}\right) \leq K \operatorname{FillVol}(M, \lambda,[\tilde{g}]),
$$

the last inequality holding because the conformal filling volume is an infimum over admissible functions that are continuous, whereas the $\tilde{\rho}$-modulus is an infimum over Borel functions.

\section{Appendix}

The proofs here closely follow the analogous ones in [32] and are included to provide the details for adapting them to the present setting.

Proof of Proposition 1.3. The zero function is admissible for the empty set, so $\mathscr{M}_{\rho}(\emptyset)=0$.

If $\mathscr{A}_{1} \subset \mathscr{A}_{2}$, then $\mathscr{F}_{\rho}\left(\mathscr{A}_{1}\right) \supset \mathscr{F}_{\rho}\left(\mathscr{A}_{2}\right)$, hence $\mathscr{M}_{\rho}\left(A_{1}\right) \leq \mathscr{M}_{\rho}\left(A_{2}\right)$.

For $\left\{\mathscr{A}_{j} \subset \Omega(X)\right\}_{j \in \mathbf{N}}$, then $\mathscr{M}_{\rho}\left(\cup_{j \in \mathbf{N}} \mathscr{A}_{j}\right) \leq \sum_{j \in \mathbf{N}} \mathscr{M}_{\rho}\left(\mathscr{A}_{j}\right)$ as follows. If $\mathscr{M}_{\rho}\left(\mathscr{A}_{j}\right)=$ $\infty$ for some $j \in \mathbf{N}$, or if the sum is not finite, then the claim is clear. Otherwise, let $\varepsilon>0$ be given. Take $f_{j} \in \mathscr{F}_{\rho}\left(\mathscr{A}_{j}\right)$ such that $\left\|f_{j}\right\|_{n}^{n}<\mathscr{M}_{\rho}\left(\mathscr{A}_{j}\right)+2^{-j} \varepsilon$. Let $\mathscr{A}=\cup_{j \in \mathbf{N}} \mathscr{A}_{j}$ and consider the function

$$
f=\left(\sum_{j \in \mathbf{N}} f_{j}^{n}\right)^{1 / n} .
$$

Since $f \geq f_{j}$ for all $j \in \mathbf{N}, f \in \mathscr{F}_{\rho}\left(\mathscr{A}_{j}\right)$ for all $j$, whence $f \in \mathscr{F}_{\rho}(\mathscr{A})$. Thus,

$$
\mathscr{M}_{\rho}(\mathscr{A}) \leq\|f\|_{n}^{n}=\sum_{j \in \mathbf{N}}\left\|f_{j}\right\|_{n}^{n}<\varepsilon+\sum_{j \in \mathbf{N}} \mathscr{M}_{\rho}\left(\mathscr{A}_{j}\right)
$$

where $\varepsilon$ is arbitrary.

Proof of Proposition 1.4. By Proposition 1.3 (ii), $\mathscr{M}_{\rho}(\mathscr{A}) \geq \mathscr{M}_{\rho}(\mathscr{B})$ since $\mathscr{A} \supset$ $\mathscr{B}$. Now we show $\mathscr{M}_{\rho}(\mathscr{A}) \leq \mathscr{M}_{\rho}(\mathscr{B})$. Either $\mathscr{M}_{\rho}(\mathscr{B})=\infty$ and we are done, or else 
we can take $f \in \mathscr{F}_{\rho}(\mathscr{B})$ with $\|f\|_{n}<\infty$. Fix $F \in L^{n}(X)$ a strictly positive function, and let

$$
f_{\varepsilon}=\left(f^{n}+\varepsilon F^{n}\right)^{1 / n} .
$$

Since $f_{\varepsilon} \geq f$, for all $\gamma \in \mathscr{B}$,

$$
\int_{\gamma} f_{\varepsilon} \geq \int_{\gamma} f \geq \rho(\gamma)
$$

If $\rho(\gamma)=0$, then $\int_{\gamma} f_{\varepsilon} \geq 0$. If $\gamma$ is not rectifiable, then

$$
\int_{\gamma} f_{\varepsilon} \geq \int_{\gamma} \varepsilon F=\infty \geq \rho(\gamma)
$$

Therefore $f_{\varepsilon} \in \mathscr{F}_{\rho}(\mathscr{A})$ and $\mathscr{M}_{\rho}(\mathscr{A}) \leq\left\|f_{\varepsilon}\right\|_{n}^{n}=\|f\|_{n}^{n}+\varepsilon^{n}\|F\|_{n}^{n}$. Since $\varepsilon>0$ and $f \in \mathscr{F}_{\rho}(\mathscr{A})$ were arbitrary, it follows that $\mathscr{M}_{\rho}(\mathscr{A}) \leq \mathscr{M}_{\rho}(\mathscr{B})$.

Proof of Proposition 1.6. Both (i) and (ii) are immediate from the definition of the inner and outer $\rho$-dilation, and (iii) follows immediately from them. If either $K_{\rho_{1}, I}(\varphi)$ or $K_{\rho_{2}, I}(\psi)$ are infinite $(i v)$ holds, so we assume they are both finite. In this case, if $\mathscr{M}_{\rho_{2}}(\varphi(\mathscr{A}))=0$, then $\mathscr{M}_{\rho_{3}}(\psi \circ \varphi(\mathscr{A}))=0$ - a superfluous case - as otherwise $K_{\rho_{2}, I}(\psi)$ would be infinite. Similarly, if $\mathscr{M}_{\rho_{1}}(\mathscr{A})=0$, then $\mathscr{M}_{\rho_{2}}(\varphi(\mathscr{A}))=0$ as otherwise $K_{\rho_{1}, I}(\varphi)$ would be infinite. Using the same argument, if $\mathscr{M}_{\rho_{2}}(\varphi(\mathscr{A}))=\infty$, then $\mathscr{M}_{\rho_{1}}(\mathscr{A})=\infty$, another case that does not affect the $\rho_{j}$-dilation. So we only need to consider the case that $\mathscr{A} \subset \Omega\left(X_{1}\right)$ and $0<\mathscr{M}_{\rho_{2}}(\varphi(\mathscr{A}))<\infty$, whence

$$
\frac{\mathscr{M}_{\rho_{3}}(\psi \circ \varphi(\mathscr{A}))}{\mathscr{M}_{\rho_{1}}(\mathscr{A})}=\frac{\mathscr{M}_{\rho_{3}}(\psi \circ \varphi(\mathscr{A}))}{\mathscr{M}_{\rho_{2}}(\varphi(\mathscr{A}))} \frac{\mathscr{M}_{\rho_{2}}(\varphi(\mathscr{A}))}{\mathscr{M}_{\rho_{1}}(\mathscr{A})} \leq K_{\rho, I}(\psi) K_{\rho, I}(\varphi)
$$

from which (iv) follows; (v) is proved similarly, and (vi) follows immediately from them.

Proof of Lemma 1.7. Since $\varphi$ is quasiconformal, there exists a sequence $\left\{\varphi_{i}\right\} \subset$ $\mathscr{C}^{1}(M, \tilde{M})$ such that $\varphi_{j} \rightarrow \varphi$ uniformly on compact subsets of $M$ and such that $\varphi_{j} \rightarrow \varphi$ in $W_{1}^{n}(U, \tilde{M})$ on pre-compact $U \subset M$. The proof of this closely follows that of Theorem 3.3 in Chapter 2 of [21] combined with Theorem 27.7 and Remark 2 of paragraph 34.8 in [32]. By Proposition 1.4 we can assume that every curve $\gamma \in \mathscr{A}$ is rectifiable and $\rho(\gamma)>0$. Let $\left\{U_{j}\right\}_{j \in \mathbf{N}}$ be an exhaustion of $X$ by open, pre-compact sets whose closures are in $X$ with $U_{j} \subset U_{j+1}$ for all $j \in \mathbf{N}$. Denote by $\mathscr{A}_{j} \subset \mathscr{A}$ all curves whose images are in $U_{j}$. For any $\gamma \in \mathscr{A}$, there exists a $j \in \mathbf{N}$ such that $\gamma \in \mathscr{A}_{j}$ whence $\mathscr{M}_{\rho}(\mathscr{A}) \leq \sum_{j \in \mathbf{N}} \mathscr{M}_{\rho}\left(\mathscr{A}_{j}\right)$ by Proposition 1.3 (iii). Therefore, it is sufficient to prove that $\mathscr{M}_{\rho}\left(\mathscr{A}_{j}\right)=0$ for all $j \in \mathbf{N}$.

Now fix $j \in \mathbf{N}$. Let $f_{i}=\left\|D \varphi_{i}-D \varphi\right\|$ be the function given by the pointwisedefined operator norm. After passing to a subsequence, we can assume that

$$
\int_{U_{j}} f_{i}^{n}<2^{-i(n+1)}
$$

Let $\mathscr{B}$ be all rectifiable paths $\gamma \in \Omega\left(U_{j}\right)$ such that $\limsup _{i \rightarrow \infty} \int_{\gamma} f_{i}>0$. Take

$$
\mathscr{B}_{i}=\left\{\gamma \in \mathscr{B} \mid \int_{\gamma} f_{i}>2^{-i} \rho(\gamma)\right\}
$$


Then $\mathscr{B} \subset \bigcup_{i \geq N} \mathscr{B}_{i}$ for any $N \in \mathbf{N}$. Also, $2^{i} \chi_{U_{j}} f_{i} \in \mathscr{F}_{\rho}\left(\mathscr{B}_{i}\right)$, and so by (3.14),

$$
\mathscr{M}_{\rho}\left(\mathscr{B}_{i}\right) \leq 2^{n i} \int_{U_{j}} f_{i}^{n}<2^{-i}
$$

Therefore, $\mathscr{M}_{\rho}(\mathscr{B}) \leq \sum_{i \geq N} \mathscr{M}_{\rho}\left(\mathscr{B}_{i}\right)<2^{-N+1}$ so $\mathscr{M}_{\rho}(\mathscr{B})=0$ since $N$ was arbitary.

We will show that $\mathscr{A}_{j} \subset \mathscr{B}$, proving the theorem. We have

$$
\int_{\gamma}\left\|\varphi_{i *} Z-\varphi_{*} Z\right\|_{\tilde{g}} \leq \int_{\gamma}\|Z\|_{g} f_{i}
$$

for any continuous vector field $Z$ along $\gamma$. When $\gamma \in \Omega\left(U_{j}\right) \backslash \mathscr{B}$ is rectifiable, the right-hand side of (3.15) converges to zero independent of $Z$. Let $\bar{\gamma}:[0, \ell] \rightarrow U_{j}$ be the re-parameterization of $\gamma$ by arclength, hence $\bar{\gamma}^{\prime}(t)$ is defined and has unit length for almost every $t \in[0, \ell]$. Since $\gamma$ is rectifiable and $\varphi_{i}$ is $\mathscr{C}^{1}, \varphi_{i} \circ \bar{\gamma}$ is absolutely continuous. Therefore for $0 \leq t_{1}<t_{2} \leq \ell$,

$$
\mathrm{d}_{\tilde{g}}\left(\varphi_{i}\left(\bar{\gamma}\left(t_{1}\right)\right), \varphi_{i}\left(\bar{\gamma}\left(t_{2}\right)\right)\right) \leq \int_{t_{1}}^{t_{2}}\left\|\varphi_{i *}\left(\bar{\gamma}^{\prime}(t)\right)\right\|_{\tilde{g}} d t \leq \int_{t_{1}}^{t_{2}}\left\|D \varphi_{i}\right\|(\bar{\gamma}(t)) d t .
$$

For $\gamma \notin \mathscr{B}$, since $\varphi_{i} \rightarrow \varphi$ uniformly on $\overline{U_{j}}$, in the limit as $i \rightarrow \infty$,

$$
\mathrm{d}_{\tilde{g}}\left(\varphi\left(\bar{\gamma}\left(t_{1}\right)\right), \varphi\left(\bar{\gamma}\left(t_{2}\right)\right)\right) \leq 2 \int_{t_{1}}^{t_{2}}\left\|D \varphi_{i}\right\|(\bar{\gamma}(t)) d t
$$

for all $i \in \mathbf{N}$ sufficiently large, whence $\varphi \circ \gamma$ is absolutely continuous so $\gamma \notin \mathscr{A}_{j}$.

\section{References}

[1] Ahlfors, L. V.: Conformal invariants. - McGraw-Hill, 1973.

[2] Arcostanzo, M.: Des métriques finslériennes sur le disque à partir d'une fonction distance entre les points du bord. - Comment. Math. Helv. 69, 1994, 229-248.

[3] Astala, K.: Area distortion of quasiconformal mappings. - Acta Math. 173, 1994, 37-60.

[4] Bangert, V., C. Croke, S. Ivanov, and M. Katz: Filling area conjecture and ovalless real hyperelliptic surfaces. - Geom. Funct. Anal. 15, 2005, 577-597.

[5] BAvard, C.: Inégalités isosystoliques conformes pour la bouteille de Klein. - Geom. Dedicata 27, 1988, 349-355.

[6] Bavard, C.: Inǵalités isosystoliques conformes. - Comment. Math. Helv. 67, 1992, 146-166.

[7] Berger, M.: Systoles et applications selon Gromov. - Séminaire Bourbaki, Vol. 1992/93, Astérisque 216, Exp. No. 771, 1993, 279-310.

[8] Chavel, I.: Riemannian geometry. A modern introduction. - Cambridge Univ. Press, 1993.

[9] Croke, C.: Rigidity for surfaces of non-positive curvature. - Comment. Math. Helv. 65, 1990, 150-169.

[10] Croke, C.: Rigidity and the distance between boundary points. - J. Differential Geom. 33, 1991, 445-464.

[11] Croke, C.: Rigidity theorems in Riemannian geometry. - In: Geometric Methods in Inverse Problems and PDE Control, edited by C. Croke, I. Lasiecka, G. Uhlmann, and M. Vogelius, IMA Vol. Math. Appl. 137, Springer, 2004, 47-72. 
[12] Croke, C., N.S. Dairbekov, and V.A. Sharafutdinov: Local boundary rigidity of a compact Riemannian manifold with curvature bounded above. - Trans. Amer. Math. Soc. 352, 2000, 3937-3956.

[13] Croke, C., and M. Katz: Universal volume bounds in Riemannian manifolds. - In: Surveys in differential geometry, Vol. VIII (Boston, MA, 2002), Int. Press, Somerville, MA, 2003, 109-137.

[14] Erëmenko, A., and D. H. Hamilton: On the area distortion by quasiconformal mappings. Proc. Amer. Math. Soc. 123, 1995, 2793-2797.

[15] Fuglede, B.: Extremal length and functional completion. - Acta Math. 98, 1957, 171-219.

[16] Gallot, S., D. Hulin, and J. Lafontaine: Riemannian geometry. - Springer-Verlag, 2nd edition, 1990.

[17] Gehring, F. W.: Rings and quasiconformal mappings in space. - Trans. Amer. Math. Soc. 103, 1962, 353-393.

[18] Gehring, F. W., and E. Reich: Area distortion under quasiconformal mappings. - Ann. Acad. Sci. Fenn. Ser. A I Math. 388, 1966, 1-14.

[19] Gromov, M.: Filling Riemannian manifolds. - J. Differential Geom. 18, 1983, 1-147.

[20] Heinonen, J., and P. Koskela: Quasiconformal maps in metric spaces with controlled geometry. - Acta Math. 181, 1998, 1-61.

[21] Hirsch, M. W.: Differential topology. - Springer-Verlag 1976.

[22] IWANIEC, T.: $L^{p}$-theory of quasiregular mappings. - In: Quasiconformal space mappings, Lecture Notes in Math. 1508, Springer, Berlin, 1992, 39-64.

[23] Katz, M.: Systolic geometry and topology. - Math. Surveys Monogr. 137, American Mathematical Society, 2007.

[24] Katz, N. N.: Minimal volume with length bounded below. - Differential Geom. Appl. 22, 2005, $297-313$.

[25] MichèL, R.: Sur la rigidité imposée par la longueur des géodésiques. - Invent. Math. 65, 1981, $71-83$.

[26] Mostow, G. D.: Quasi-conformal mappings in $n$-space and the rigidity of hyperbolic space forms. - IHES Sci. Publ. Math. 34, 1968, 53-104.

[27] Otal, J.-P.: Sur les longeurs des géodésiques d'une métrique à courbure négative dans le disque. - Comment. Math. Helv. 65, 1990, 334-347.

[28] Pestov, L., and G. Uhlmann: Two dimensional compact simple Riemannian manifolds are boundary distance rigid. - Ann. of Math. (2) 161, 2005, 1093-1110.

[29] Rešetnjak, Ju. G.: Stability of conformal mappings in multi-dimensional spaces. - Sibirsk. Mat. Zh. 8, 1967, 91-114.

[30] Santalo, L. A.: Integral geometry and geometric probability. - Addison-Wesley, Reading, MA, 1976.

[31] Sharafutdinov, V. A.: Integral geometry of tensor fields. - Inverse Ill-posed Probl. Ser., VSP, Utrecht, 1994.

[32] VÄıs̈̈LÄ, J.: Lectures on n-dimensional quasiconformal mappings. - Lecture Notes in Math. 229, Springer-Verlag, 1971. 